\title{
REPRODUCTION OF THE LYMAN $\alpha$ IRRADIANCE VARIABILITY FROM ANALYSIS \\ OF FULL-DISK IMAGES IN THE CaII K-LINE
}

\author{
A. JOHANNESSON, W. MARQUETTE, and H. ZIRIN \\ Big Bear Solar Observatory, California Institute of Technology \\ Pasadena, CA 91125
}

(Received 10 April, 1995; in revised form 10 July, 1995)

\begin{abstract}
We have compared three years of daily CaII K-line images from the Big Bear Solar Observatory (BBSO) with HI Lyman $\alpha$ irradiance data from the Upper Atmosphere Research Satellite (UARS). The daily full-disk CaII K-line images are reduced to a new index of integrated excess emission, which reproduces both the 27 day rotational modulation and the solar cycle decrease in Ly $\alpha$ irradiance. Our analysis shows that while plages reproduce the 27-day variation quite well, the total K-line emission excess above the quiet background is needed to reproduce the secular solar cycle trend in the Ly $\alpha$ irradiance. The resulting K-line index exhibits a high degree of correlation (0.9) with the time series of measured Ly $\alpha$ flux.
\end{abstract}

\section{Introduction}

Spatially resolved images of the Sun from the ground in the CaII K-line (3933 A) and from space in the Ly $\alpha$ line $(1250 \mathrm{~A}$ ) appear quite similar (Bonnet et al., 1980 ), so it is plausible that CaII K-line observations could be used to estimate the irradiance of the strongest UV line. Various measurements of CaII indices (Lean et al., 1982; Pap et al., 1991a; Pap et al., 1991b) have shown that this is possible, but that the enhanced network must be included in such measurements. CaII K-line plage indices can reproduce the rotational modulation (Donnelly et al., 1986) quite well, but they generally underestimate the longer-term variations due to the 11-year cycle (Lean, 1991). A longer, more accurate database of UV irradiance and digital $\mathrm{K}$-line measurements with resolution and contrast adequate to measure the enhanced network were needed.

The SOLSTICE and SUSIM experiments on UARS (Rottman, 1988; Reber, 1993; London et al., 1993; Brueckner et al., 1993) have provided high-quality irradiance measurements in the wavelength region 1100-4200 A since October 1991. Full-disk CaII K-line images (BBSO data) have been recorded daily at the Big Bear Solar Observatory (BBSO) since October 1981 (digitally since October 1982), with only a short break due to the 1992 earthquake. Call K-line plages were systematically measured to produce an index which was published until November 1987 when this work had to be discontinued for lack of funds. Because of the long-term significance of these observations, we continued a low-level program of simply recording and digitizing the images (Marquette, 1992) without analysis. The UARS guest investigator program made it possible to measure the BBSO data

Solar Physics 161: 201-204, 1995.

(C) 1995 Kluwer Academic Publishers. Printed in Belgium. 
and compare it with the new long database of accurate Ly $\alpha$ irradiance measurements, and the digitized data made it possible to evaluate possible contributions to the Ly $\alpha$ irradiance.

\section{BBSO CaII K-line Data and Data Reduction}

The BBSO data are taken with a Daystar 1.0 A filter centered on the CaII K-line at $3933 \mathrm{~A}$. We currently use a Cohu video camera and an 8-bit Aspro digitizer to obtain the full-disk CaII $\mathrm{K}$-line images. The scale of the digitized images is 4 arc seconds per pixel.

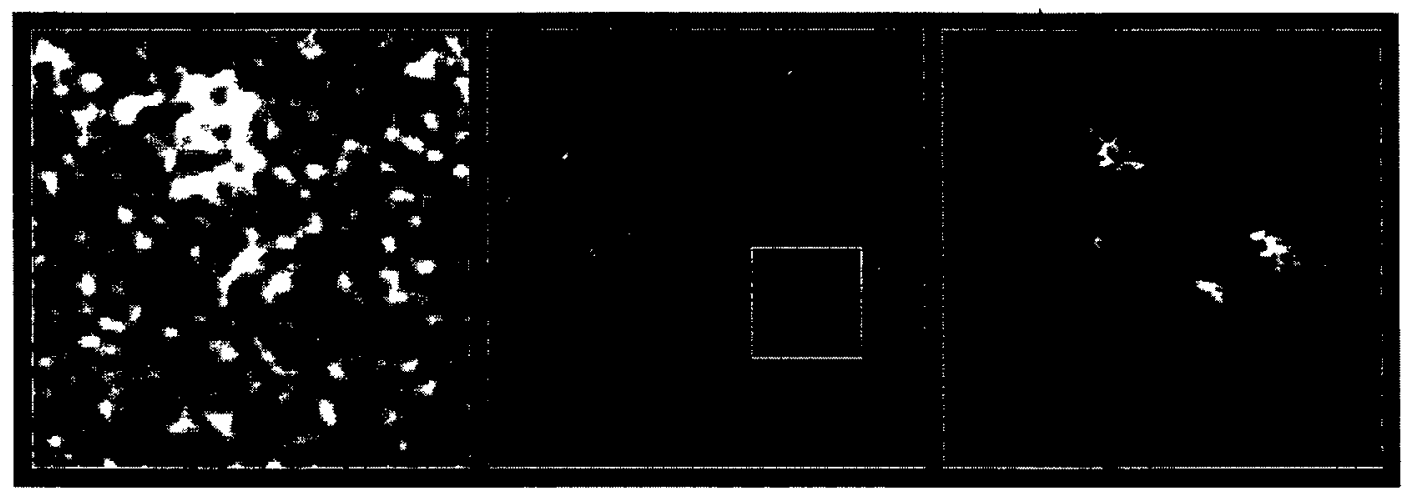

Fig. 1. CalI K-line images (reduced for limb darkening) showing the Sun with different activity features (Aug. 2 [middle] and Sep. 7 [right], 1994) producing the same UARS Ly $\alpha$ irradiance and about the same K-line indices. To the left is a detail of the Aug. 2 image which displays the CaII $\mathrm{K}$-line network present in the BBSO images.

The earlier digital data had not been analyzed so there had been no quality feedback, and images were somewhat irregular and variable. Since mid-year 1994, a fixed black level has been used, and the gain is more uniform. As a result, the quality of results of the image analysis have improved.

We used an IDL routine to remove the effects of limb darkening and instrumental non-uniformity. Because the software cannot assume a symmetrical limb darkening function, the quiet-sun brightness is estimated in each image from the areas between bright magnetic features. The gain is adjusted for constant contrast of quiet-sun features. Bright magnetic features are found by a combination of an edge detector and a median filter. The pixels closest to the limb are treated separately using the assumption that solar activity and the quiet-sun background have well-separated spatial frequencies in circular tracings at large radii.

We subtract the estimated background from the original image to get a residual image without limb darkening or brightness enhancements (Fig. 1). An activity index is then calculated as:

$$
\text { Index }=c \sum_{i j} f_{i j}+m \quad f_{i j}= \begin{cases}R_{i j}^{p} & \text { if } R_{i j}>T \\ 0 & \text { if } R_{i j} \leq T\end{cases}
$$


where $R$ is the residual image, $T$ is a threshhold, and $c$ and $m$ are the coefficients of a fit to the UARS data. $T$ is set to $2 \sigma$ above the background. By setting $T$ and the free parameter $p$ we found the best fit to the data with $p=0.5$. Because we do not have spatially resolved photometric Ly $\alpha$ data, we do not know the contribution from individual features.

\section{Results}

We analyzed 750 CaII K-line images which represent the part of the BBSO database covering the available UARS Ly $\alpha$ data (October 1991 - September 1994). The comparison (Fig. 2) between the UARS SOLSTICE Ly $\alpha$ irradiance (London $e t$ al., 1993) and the BBSO index shows a correlation coefficient of 0.9 for the whole period, 1991 to 1994, with no phase lag. The correlation is somewhat lower after a detrend ( 0.8 for 1994). Due to the combination of a lack of dynamic range in the digital images and low frequency flat fielding errors, we cannot accurately measure the network features with excess brightness below $3 \%$ of the disk center intensity.

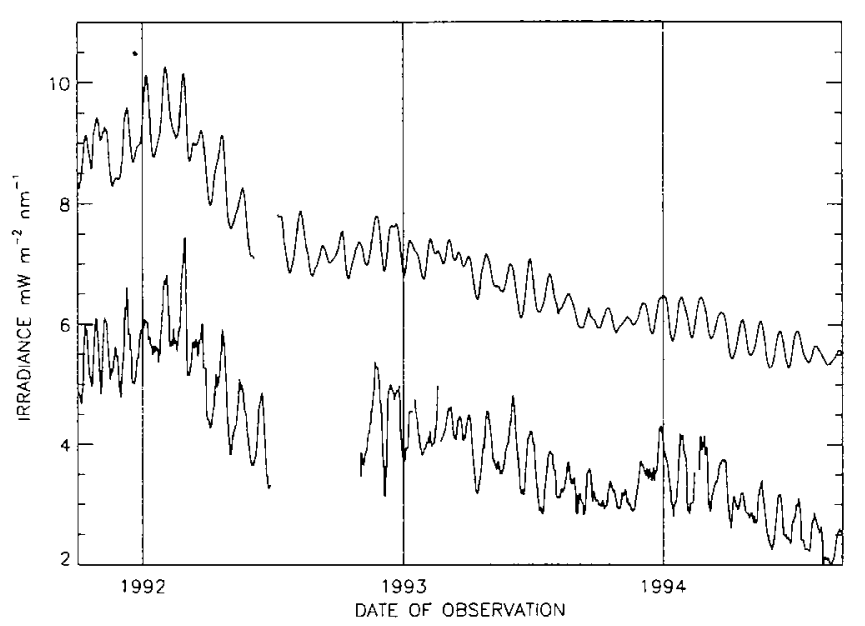

Fig. 2. UARS SOLSTICE HI Lyman $\alpha$ irradiance (upper graph) and BBSO CaII K-line index (lower graph). The BBSO data have been fitted to the UARS plot by linear regression. Both plots are seven-day running averages. The gap in 1992 is due to an earthquake.

Both data sets are filtered with a seven-day running average. The BBSO data are fitted to the UARS data by linear regression and is shifted for clarity. Notice that both the short-term modulation, mostly due to the rotation of plages, and the long-term decrease, due to the overall decrease in solar activity, are fairly well represented in the BBSO data. If the network is omitted the long-term trend cannot be fitted. 
There are exceptions to the good time correlation. At the end of 1992 and the middle of 1993, the BBSO data have about twice the amplitude of rotational modulation of the UARS SOLSTICE Ly $\alpha$ irradiance. At this point we cannot distinguish real differences from the effects of data gaps and gain variations.

The excess K-line emission is due to a range of sources. Fig. 1 shows two quite different distributions that give the same resulting $\mathrm{K}$-line and Ly $\alpha$ indices. Thus it seems that there are no special sources, all excess $\mathrm{K}$-line was measured equally. Fig. 2 shows that the Ly $\alpha$ variation can not be explained without the contribution of the widespread Call K-line network. The good fit of the Ly $\alpha$ variation by the $\mathrm{K}$-line excess suggests that the background Sun does not change with the cycle.

\section{Conclusion}

We have empirically fitted the Ly $\alpha$ irradiance measured by the UARS satellite using ground-based CaII K-line images from BBSO. By including everything above $2 \sigma$ above the background, we can fairly well reproduce the rotational modulation as well as the secular decline at the end of Solar Cycle 22. We plan to expand the comparison to other UARS UV wavelengths and to BBSO H $\alpha$ images. We have recently ordered a digital CCD camera, which will provide much higher photometric quality and stability.

\section{Acknowledgements}

The SOLSTICE and SUSIM data were provided courtesy of the NASA UARS project by Drs. Gary Rottman and Guenter Brueckner, respectively. We thank Dr. Judit Pap and Dr. Oran White for helpful discussions. This research is funded by the UARS Guest Investigater Program of NASA under grant NAG5-2782.

\section{References}

Bonnet, R. M., Bruner, E. C.. Acton. L. W., Brown, W. A., Decaudin, M.: 1980, Astrophys.J. 237, L47.

Brueckner. G. E.. Edlow. K. L.. Floyd. L. E., Lean, J. L. and VanHoosier, M. E.: 1993, Journal of Geophys. Res. 98, 695.

Donnelly, R. F., Puga, L. C., and Busby, W. S.: 1986. NOAA Technical Memorandum ERL ARL-146

Lean, J.: 1991, Revs. of Geophys. 29, 505.

Lean, J. et al.: 1982, J. Geophys. Res. 87, 307.

London, J., Rottman, G. J.. Woods, T. N.: 1993, Geophys. Res. Let. 20, 1315.

Marquette, W. H.: 1992, in the Proceedings of the Workshop on the Solar Electromagnetic Radiation Study for Solar Crcle 22.

Pap, J. M., London, J., Rottman. G. J.: 1991 a, Astron. Astrophys. 245, 648.

Pap, J. M., Marquette. W. H., Donnelly, R. F.: 199lb, Adv. Space Res. 8, 271.

Reber, C. A.: 1993, Geophys. Res. Let. 20, 1215.

Rottman, G. J.: 1988, Adv. Space Res. 8, 31. 